\title{
Theoretical and experimental investigation of parametrically excited piezoelectric energy harvester
}

\author{
Anshul Garg ${ }^{1, *}$ and Santosha K. Dwivedy ${ }^{1}$ \\ ${ }^{1}$ Department of Mechanical Engineering, IIT Guwahati, Guwahati, 781039, Assam, India
}

\begin{abstract}
In the present work, a cantilever beam based piezoelectric energy harvester is investigated both theoretically and experimentally. The harvester is consists of a harmonically base excited vertical cantilever beam with a piezoelectric patch at the fixed end and a mass attached at an arbitrary position. The Euler-Bernoulli beam theory is applied considering the cantilever beam to be slender. The temporal nonlinear electromechanical governing equation of motion is obtained by using generalized Galerkin's method considering two-mode approximation. Here for principal parametric resonance condition the steady state response of the voltage is obtained by using the method of multiple scales. The results are validated by developing an experimental setup of the harvester. For the harvester having a dimension of $295 \mathrm{~mm} \times 24 \mathrm{~mm} \times 7.6 \mathrm{~mm}$, a maximum voltage of $40 \mathrm{~V}$ is obtained for a base motion of $9 \mathrm{~mm}$ with a frequency of $10.07 \mathrm{~Hz}$ when $15 \mathrm{gm}$ mass is attached at a distance of $140 \mathrm{~mm}$ from the fixed end.
\end{abstract}

\section{Introduction}

Development in smart devices used for sensing, human and structural health monitoring and actuation requires low power to operate. These devices are operated by batteries which in addition, are having a limited life span, environmentally unfriendly and requires a regular replacement. This dependency of smart devices on power supply is not really smart. So to overcome this drawback researchers are exploring other nontraditional means to power these devices by extracting untapped ambient energy from sources such as light, wind, temperature and potential gradient, noise, sound and vibration etc. This energy can be transformed by three basic transduction mechanisms namely electromagnetic, electrostatic and piezoelectric. Out of three the piezoelectric transduction mechanism is getting the most attention due to its high power density and ease of application [10].

In general, linear vibration based piezoelectric energy harvester systems work over a short range of bandwidth near the resonance frequency. Energy transduction reduces sharply for mistuned (away from resonance) linear harvesting systems. To address this issue researchers are focusing on tapping the rich dynamics which is outcome of inherent or induced nonlinearity. Nonlinear systems display behaviours such as bifurcation, chaos,

\footnotetext{
* Corresponding author: anshul.sv@gmail.com
} 
internal resonance that linear systems cannot. The multi-branched fixed point response of a nonlinear system may be periodic, quasi-periodic and chaotic in nature. Energy harvesters based on nonlinear vibration have potential to extract energy due to multiple resonance conditions which may lead to enhanced frequency band width. The source of nonlinearity can be of the geometric or material in nature. It can also arise due to external excitation and constraints such as impact, friction, backlash and freeplay $[4,5]$. The frequency bandwidth over which the higher transduction of mechanical energy to electrical energy takes place can be increased by exploiting the inherent or induced nonlinearity [6].

A large response can be produced by small parametric excitation [7] even when the frequency of excitation is away from the fundamental frequency of the system. Daqaq et al., [3] investigated a nonlinear parametrically excited cantilever beam based harvester. A critical amplitude of excitation is necessary to be maintained always to get nontrivial system response. In a similar work where parametric excitation is considered Abdelkefi et al., [9] developed a distributed parameter model of parametrically excited nonlinear PEH system having a cantilever beam and a tip mass.

In the present work a parametrically excited cantilever beam with piezoelectric patch and an attached mass is considered for dynamic analysis. The similar system is analyzed by Zavodney and Nayfeh [1] considering single mode approximation and without piezoelectric element. Also by considering two mode approximation similar model is analysed analytically by Kar and Dwivedy [2] and Dwivedy and Kar [8] without piezoelectric patch. Here the Generalized Galerkin's method is used to discretize the spatio-temporal equation of motion to its temporal form. Steady state response and output voltage is obtained by using perturbation method namely the Method of multiple scales (MMS) to reduce the equations of motion into first order differential equations. In house experimental setup is developed in order to verify the results obtained analytically.

\section{Mathematical modeling}

The schematic of parametrically excited $\left(z(t)=z_{0} \cos (\Omega t)\right)$ piezoelectric energy harvester of length $L$ consists of a mass $m$ at a distance $d$ and piezoelectric patch of length $L_{p}$ is shown in Fig. 1. At a distance $s, u(s, t)$ and $v(s, t)$ denotes the longitudinal and transverse displacements. The curvature and displacement expression is considered nonlinear due to large transverse geometric deflection. The following nonlinear electromechanical equation of motion $[1,2,10]$ is obtained

$$
[\rho+m \delta(s-d)] v_{t t}+c v_{t}+E I\left\{v_{s s s s}+\left[v_{s}\left(v_{s} v_{s s}\right)_{s}\right]_{s}\right\}-\left[J_{0} \delta(s-d)\left(v_{s}\right)_{t t}\right]_{s}-\left(N v_{s}\right)_{s}-\bar{H} \bar{v} \bar{V}(t)=0
$$

Here $\rho, \delta(), c, E I, J_{0}, \bar{H}, \bar{V}$ and $\bar{v}$ denotes mass per unit length, Dirac delta function, damping coefficient, flexural rigidity, moment of inertia of attached mass, Heaviside function, voltage and coupling term for series connection respectively. 


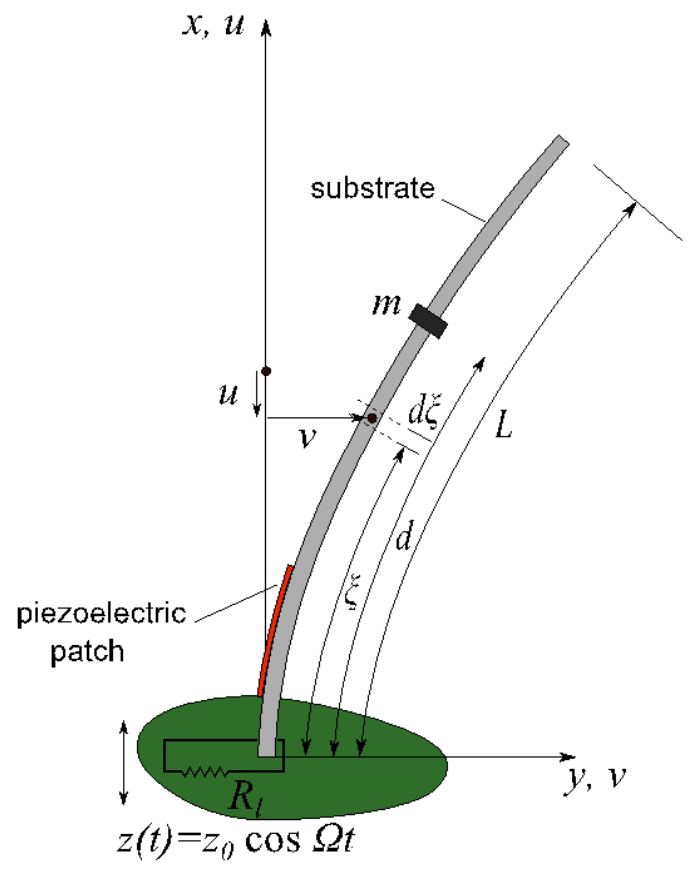

Figure 1. Schematic of a parametrically excited piezoelectric energy harvester (PEH)

The inextensibility condition $u(s, t)=(1 / 2) \int_{0}^{s}\left\{v^{\prime}(\xi, t)\right\}^{2} d \xi+z(t)$ defines the longitudinal motion in terms of lateral. The circuit equation [10] for series connection under the external load resistance, $R_{l}$ becomes

$$
\frac{C_{p}}{2} \frac{d \bar{V}(t)}{d t}+\frac{\bar{V}(t)}{R_{l}}-i_{p}(t)=0
$$

The boundary conditions are $v(0, t)=0, v_{s}(0, t)=0, v_{s s}(L, t)=0, v_{s s s}(L, t)=0$

Here $\xi, s, t, C_{p}$ and $i_{p}$ denotes the variable of integration along longitudinal direction, curvilinear variable and time, capacitance, and current source. As per Galerkin's method the transverse displacement $v(s, t)$ is represented by $v(s, t)=\sum_{n=1}^{\infty} r \psi_{n}(s) q_{n}(t)$ where $r, q_{n}(t), \psi_{n}(s)$ denotes scaling factor, time modulation and shape function. After nondimensionalization the temporal governing equations of motion becomes

$$
\begin{gathered}
\ddot{q}_{n}+2 \varepsilon \zeta_{n} \dot{q}_{n}+\omega_{n}^{2} q_{n}+\varepsilon \sum_{k, l, m}^{2}\left(\alpha_{k l m}^{n} q_{l} q_{m}+\beta_{k l m}^{n} \dot{q}_{l} \dot{q}_{m}+\gamma_{k l m}^{n} q_{l} \ddot{q}_{m}\right) q_{k}+ \\
\varepsilon v_{n} V(\tau)-\varepsilon \sum_{m=1}^{2}\left[f_{n m} \cos (\phi \tau)\right] q_{m}=0 \\
\dot{V}(\tau)+r_{e} V(\tau)+\sum_{n=1} K_{n} \dot{q}_{n}=0
\end{gathered}
$$

Here we assume that the terms $\zeta_{n}, \alpha_{k l m}^{n}, \beta_{k l m}^{n}, \gamma_{k l m}^{n}, v_{n},\left[f_{n m} \cos (\phi \tau)\right] q_{m}$ which denotes the damping, geometric and inertial nonlinearities, electromechanical coupling and parametric excitation [7] are small hence considered of the order of $\varepsilon$ (book keeping parameter). In the 
absence of piezoelectric patch the equation of motion of the system reduces to the model investigated by Zavodney and Nayfeh [1].

\section{Perturbation analysis}

To obtained analytical expression for transverse displacement, generated voltage and power, a uniform first order approximate analytical solution of Eq. (4) and (5) is obtained. Method of multiple scales (MMS) is used to meet this end which describe the dynamics of the nonlinear system. The system dynamics under parametric resonance case is studied. In order to implement MMS the time dependence is discretize into multiple time scales as $T_{i}=\varepsilon^{i} \tau ; i=0,1,2, \ldots$ and time derivatives expressed as:

$$
d / d \tau=D_{0}+\varepsilon D_{1}+O\left(\varepsilon^{2}\right), d^{2} / d \tau^{2}=D_{0}+2 \varepsilon D_{0} D_{1}+O\left(\varepsilon^{2}\right)
$$

where $D_{i}=\partial / \partial T_{i}$. The solution of displacement and voltage $\left(q_{n}, V\right)$ expressed, expanded or discretize as

$$
q_{n}(\tau ; \varepsilon)=q_{n 0}\left(T_{0}, T_{1}\right)+\varepsilon q_{n 1}\left(T_{0}, T_{1}\right)+O\left(\varepsilon^{2}\right) ; \quad V(\tau ; \varepsilon)=V_{0}\left(T_{0}, T_{1}\right)+\varepsilon V_{1}\left(T_{0}, T_{1}\right)+O\left(\varepsilon^{2}\right)
$$

Later substituting these expansions of solution (Eq. (6)) in Eq. (4) and comparing the coefficients involving terms $\varepsilon^{0}$ and $\varepsilon^{1}$, yields the following set of differential equations

$$
\begin{aligned}
& O\left(\varepsilon^{0}\right): \quad D_{0}^{2} q_{n 0}+\omega_{n}^{2} q_{n 0}=0 ; \quad D_{0} V_{0}+r_{e} V_{0}=-\sum_{n=1}^{\infty} K_{n} D_{0} q_{n 0} \\
& O\left(\varepsilon^{1}\right): \quad D_{0}^{2} q_{n 1}+\omega_{n}^{2} q_{n 1}=-\left\lfloor\begin{array}{l}
2 \xi_{n} D_{0} q_{n 0}+2 D_{0} D_{1} q_{n 0}-\sum_{n, m=1}^{\infty} f_{n m} q_{m 0} \cos \phi \tau+v_{n} V_{0} \\
+\sum_{k l m}\left(\alpha_{k l m}^{n} q_{k 0} q_{l 0} q_{m 0}+\beta_{k l m}^{n} q_{k 0} D_{0} q_{l 0} D_{0} q_{m 0}+\gamma_{k l m}^{n} q_{k 0} q_{l 0} D_{0}^{2} q_{m 0}\right)
\end{array}\right\rfloor \\
& D_{0} V_{1}+r_{e} V_{1}=-D_{0} V_{0}-K_{1}\left(D_{0} q_{11}+D_{1} q_{10}\right)-K_{2}\left(D_{0} q_{21}+D_{1} q_{20}\right)
\end{aligned}
$$

The solution of Eq. (7) expressed in the following form

$$
q_{n 0}=A_{n}\left(T_{1}\right) \exp \left(i \omega_{n} T_{0}\right)+c c, \quad V_{0}=-\sum_{n=1}^{2} K_{n} Z_{n} A_{n}\left(T_{1}\right) \exp \left(i \omega_{n} T_{0}\right)+c c
$$

Here $A_{n}\left(T_{1}\right)$ is a slowly varying complex valued function and $c c$ is an abbreviation for complex conjugate term. Further expanding Eq. (8) by utilizing Eq. (9) the secular and near secular terms are obtained for first two modes $(n=1,2)$. These obtained secular terms should be eliminated so that finite amplitude response exist.

\section{Case : Principal parametric excitation $\left(\phi=2 \omega_{1}+\varepsilon \sigma_{1}\right)$}

Considering only single mode approximation $(n=1)$ and when excitation frequency $(\phi)$ is near to twice of first natural frequency of the system that is $\phi \approx 2 \omega_{1}$ (condition of principal parametric excitation). After solving the electromechanical equation of motion by Method of multiple scales and invoking the solvability condition by making secular term zero to find the value of unknown $A_{1}\left(T_{1}\right)$. Writing $\phi=2 \omega_{1}+\varepsilon \sigma_{1}$ where $\sigma_{1}$ and $\varepsilon$ are detuning and book keeping parameter which shows the nearness of excitation frequency to $2 \omega_{1}$.

$$
e^{I T_{0} \omega_{1}}\left(-Z_{1} \nu_{\bar{s}} A_{1}-2 I \zeta_{1} \omega_{1} A_{1}-\left(3 \alpha_{111}+\beta_{111} \omega_{1}^{2}-3 \gamma_{111} \omega_{1}^{2}\right) A_{1}^{2} \bar{A}_{1}-2 I \omega_{1} A_{1}^{\prime}\right)+\frac{1}{2} \mathrm{e}^{I\left(\phi-\omega_{1}\right) T_{0}} f_{11} \bar{A}_{1}
$$


Letting $A_{1}=\frac{1}{2} a_{1}\left(T_{1}\right) \exp ^{I \beta_{1}\left(T_{1}\right)}$ and expanding secular term (coefficient of $e^{I \omega_{1} T_{0}}$ ) then separating the real and imaginary parts the reduced modulation equations at steady state $\left(a_{1}^{\prime}=\delta^{\prime}=0\right)$ becomes

$$
\sigma_{1}=\left(\frac{a_{1}^{2}}{8 \omega_{1}}\left(3 \alpha_{111}+\left(\beta_{111}-3 \gamma_{111}\right) \omega_{1}^{2}\right)+\frac{v_{\bar{s}} \omega_{1}}{2\left(r_{e}^{2}+\omega_{1}^{2}\right)}\right) \pm \sqrt{\left(\frac{f_{11}}{4 \omega_{1}}\right)^{2}-\left(\zeta_{1}+\frac{v_{\bar{s}} r_{e}}{2\left(r_{e}^{2}+\omega_{1}^{2}\right)}\right)^{2}}
$$

Here $\delta=-\beta_{1}+\sigma_{1} T_{1}$ and $\beta_{1}^{\prime}=-\delta^{\prime}+\sigma_{1}$.

Table 1. Properties of Piezoelectric Patch SP-5H (PZT-5H) [11]

\begin{tabular}{lll} 
Property & Sym & Value \\
\hline Dielectric constant & $K_{33}^{T}$ & 3250 \\
Capacitance, $\mathrm{nF}$ & $C_{p}$ & 71.3034 \\
Piezo constant, $\mathrm{CN}^{-1}$ & $d_{31}$ & $-285 \times 10^{-12}$ \\
\hline
\end{tabular}

Table 2. Geometric and material properties.

\begin{tabular}{lll} 
Property & Piezoelectric & Substrate \\
\hline Young's Moduli, GPa & $E_{p}=66.7$ & $E_{b}=190$ \\
Density, $\mathrm{kg} \mathrm{m}^{-3}$ & $\rho_{p}=7500$ & $\rho_{b}=5555$ \\
Length, m & $L_{p}=5 \times 10^{-2}$ & $L_{b}=29.5 \times 10^{-2}$ \\
Width, $\mathrm{m}$ & $b_{p}=2.4 \times 10^{-2}$ & $b_{b}=2.4 \times 10^{-2}$ \\
Height, $\mathrm{m}$ & $t_{p}=5 \times 10^{-4}$ & $t_{b}=7.6 \times 10^{-4}$ \\
\hline
\end{tabular}
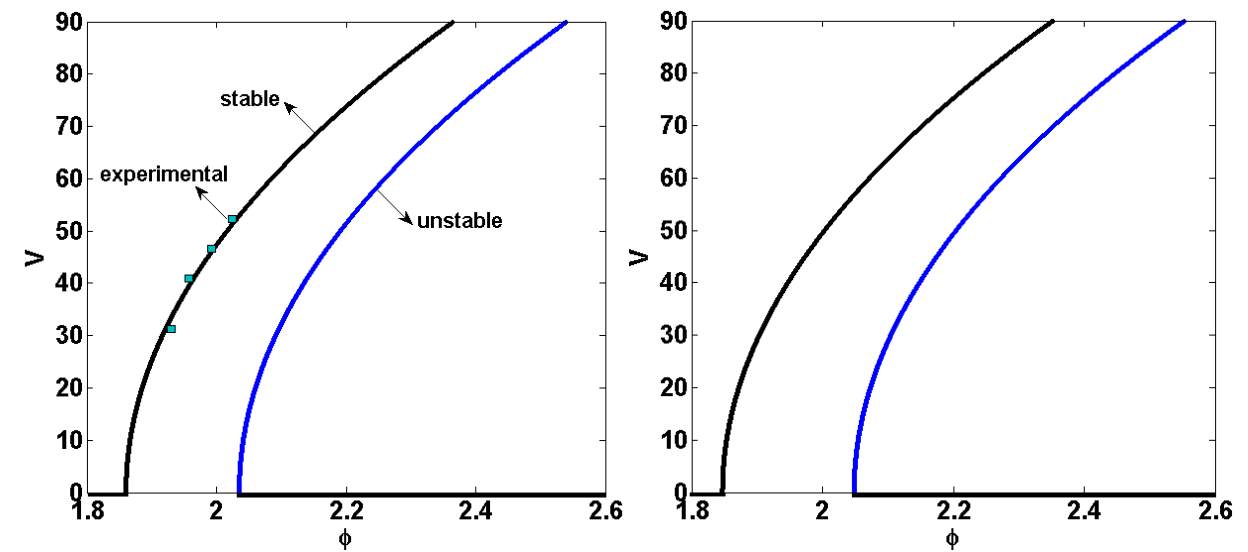

Figure 2. Voltage variation with excitation frequency for $v=0.005$ and $v=1$

The variation of voltage with nondimensional frequency of excitation is shown in Fig. 2 for two different parameter of damping. For higher damping the bandwidth increases slightly. The experimental findings (see Fig. 2) matches with analytical analysis. A voltage range from $30 \mathrm{~V}$ to $50 \mathrm{~V}$ is obtained when the system excited near to parametric excitation. The voltage follows the amplitude topologically due to proportionality.

\section{Experimental setup}

In house experimental setup consists of mbed NXP LPC1768 Microcontroller, encoder, display unit, power supply, PM DC motor (24 V, $3000 \mathrm{rpm})$, energy harvester etc., as shown in Fig. (3). Output voltage is obtained using the oscilloscope (InfiniiVision DSO-X$3024 \mathrm{~A}$ ). The harvester consists of a $50 \mathrm{~mm}$ long and $24 \mathrm{~mm}$ wide PZT patch attached to a stainless steel beam with a $15 \mathrm{~g}$ mass attached at a distance of $140 \mathrm{~mm}$. The cantilever beam is having dimensions of $295 \mathrm{~mm} \times 24 \mathrm{~mm} \times 7.6 \mathrm{~mm}$. The properties of the PZT and beam 
can be found in Table 1 and Table 2. The developed shaker is used to excite the beam near twice its first natural frequency which is termed as parametric excitation. The frequency range of the shaker is $25 \mathrm{~Hz}$ and stroke length is $9 \mathrm{~mm}$.

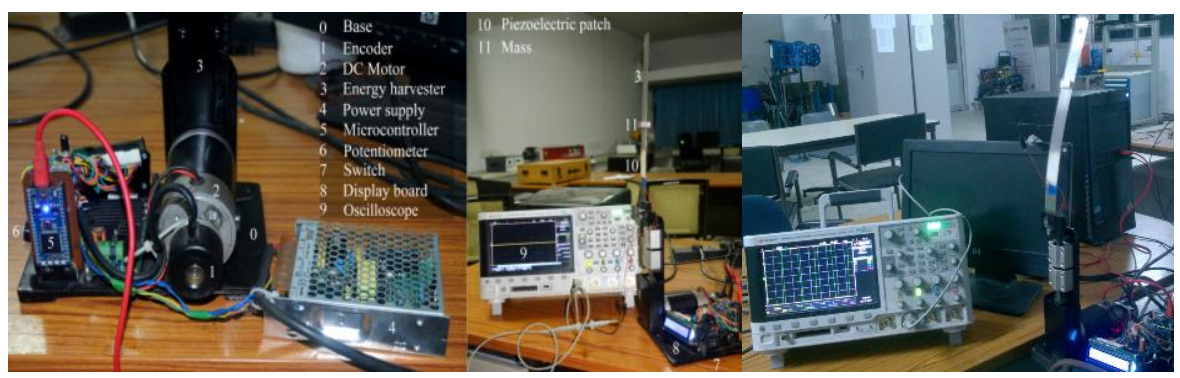

Figure 3. Experimental setup with all components and voltage response of PEH system under parametric excitation

\section{Conclusion}

A parametrically excited harvesting system consists of a cantilever beam with piezoelectric patch and attached mass is analysed. Analytical expressions are developed to measure the steady-state amplitude and voltage for parametric excitation case. Method of multiple scales is used to obtain reduced expressions which are compared with experimental findings and found to be in good agreement. High voltage of approximately $40 \mathrm{~V}$ is obtained. One can vary the mass and its position along the beam to adjust the frequency of the system accordingly to the frequency of excitation. This analysis is limited to parametric excitation but one may analyse the system by considering multimode dynamics with internal resonance.

\section{References}

[1] L.D. Zavodney, A.H. Nayfeh, Int. J. Non-Linear Mech. 24(2), 105-125 (1989)

[2] R.C. Kar, S.K. Dwivedy, Int. J. Non-Linear Mech. 34(3), 515-529 (1999)

[3] M.F. Daqaq, C. Stabler, Y. Qaroush, T. Seuaciuc-Osório, J. Intel. Mat. Syst. Str. 20(5), 545-557 (2009)

[4] J.J. Thomsen, Vibrations and stability: Advanced theory, analysis, and tools (Springer, Berlin, 2003)

[5] D. Wagg, S. Neild, Nonlinear vibration with control (Springer-Verlag, 2009)

[6] M. F. Daqaq, R. Masana, A. Erturk, D. Dane Quinn, Appl. Mech. Rev. 66, 040801 (2014)

[7] A. H. Nayfeh, D. T. Mook, Nonlinear oscillations (John Wiley \& Sons, 2008)

[8] S.K. Dwivedy, R.C. Kar, J. Sound Vib., 222(2), 281-305 (1999)

[9] A. Abdelkefi, A.H. Nayfeh, M.R. Hajj, Nonlinear Dyn., 67(2), 1147-1160 (2012)

[10] A. Erturk, D.J. Inman, Piezoelectric energy harvesting (John Wiley \& Sons, 2011)

[11] Sparkler Ceramics, Pvt. Ltd., J-508, MIDC, Bhosari, Pune-411 026, India 